\title{
Factors Affecting Non-Insulin Antidiabetic Drug Adherence in Patients with Type 2 Diabetes: A Systematic Review
}

\author{
Lindsay Petrenchik and F Ellen Loh* \\ Department of Social, Behavioral and Administrative Sciences, Touro College of Pharmacy, USA
}

Submission: February 08, 2020; Published: March 05, 2020

*Corresponding author: F Ellen Loh, Department of Social, Behavioral and Administrative Sciences Touro College of Pharmacy, 2090 Adam Clayton Powell Jr. Blvd, New York, 10027, USA

\begin{abstract}
Objective: To identify factors associated with medication non-adherence in patients with type 2 diabetes using non-insulin anti-diabetic medication.

Methods: PubMed, EMBASE/MEDLINE, and Cochrane Library databases were searched from January to March 2017. Studies were included if they used medication possession ratio (MPR) or proportion of days covered (PDC) to evaluate adherence associated with factors, were published in the last 10 years, and did not include insulin in the adherence calculations. The factors were grouped based on the five dimensions previously shown to affect medication adherence as defined by the World Health Organization.

Results: Of the 25 articles included in the review, the major factors reported to be associated with non-insulin anti-diabetic medication were younger age, female, non-Caucasian race, not using mailed prescriptions, cancer diagnosis, lower number of comorbidities, and a smaller pill burden.

Conclusion: The systematic review showed that younger age, racial minorities, female gender, fewer comorbidities, diagnosis of cancer and decreased pill burden is associated with medication nonadherence to non-insulin anti-diabetic drugs.

Keywords: Medication adherence; Non-insulin antidiabetic drugs; Type 2 diabetes; Medication possession; Glucagon-like peptide-1; General practitioner; Healthcare providers

Abbrevations: T2DM: Type 2 Diabetes Mellitus; NIAD: Non-Insulin Anti-Diabetic Drugs; MNA: Medication Nonadherence; WHO: World Health Organization, SR: Systematic Review; MPR: Medication Possession Ratio; PDC: Proportion of Days Covered, PQA: Pharmacy Quality Alliance;
\end{abstract} GLP1: Glucagon-Like Peptide-1; NIH: National Institute of Health; GP: General Practitioner; HCPs: Healthcare Providers

\section{Introduction}

As of 2014, there were 29.1 million Americans diagnosed with diabetes mellitus and it is estimated that type 2 diabetes mellitus (T2DM) accounts for 90 to $95 \%$ of these cases [1]. Approximately $70 \%$ of patients with diabetes control their glucose with a non-insulin anti-diabetic drugs (NIAD) [2]. Glycemic control is important because it decreases the risk of micro- and macrovascular complications, as well as all-cause mortality [3]. However, glycemic control is achieved in only about $50 \%$ of patients, with the primary cause being non-adherance [4]. It is estimated that only between $30.4 \%$ to $70.6 \%$ of people using NIAD are adherent to their medication [5]. It has been found that when patients are adherent to metformin alone, the probability of achieving glycemic control is up to $28 \%$ higher [6].
Understanding the factors associated with Medication Non Adherence (MNA) is essential for developing interventions to increase adherence. The World Health Organization (WHO) describes adherence as an interplay of five dimensions, which include: social and economic factors, health care team and system related factors, condition-related factors, therapy related factors, and patient related factors [7,8]. WHO explains that many interventions to increase adherence fail because there is a tendency to focus only on one of the listed factors. Therefore, it is important to understand and quantify the specific factors associated with MNA in diabetes in order to design more effective interventions [7]. There have been numerous studies evaluating associations with MNA in patients with T2DM. The most notable 
systematic review (SR) conducted by the WHO found the factors that most negatively contributed to MNA were high cost of care, concurrent depression, long duration of the disease, and complex treatment [7]. However, there has not yet been a SR to quantify the effect of factors associated with MNA. To help address this information gap, this review aimed to quantify the effect of factors associated with MNA in patients with T2DM using NIAD.

\section{Methods}

\section{Search methods for identification of studies}

A systematic search of electronic databases was conducted to identify observational studies that evaluated factors associated with adherence to anti-diabetic medication in T2DM patients. The following databases were searched: PubMed, EMBASE/MEDLINE, and Cochrane Library. The search was conducted between January $29^{\text {th }} 2017$ and March $23^{\text {rd }} 2017$. The search filters included: full text, English language, and published from January 2007 and onwards. The data was quantified using the two most common methods to measure adherence: medication possession ratio (MPR) and proportion of days covered (PDC). The key terms 'proportion of days covered' and 'medication possession ratio' were combined with the medical subject headings "diabetes mellitus, type 2 " and "medication adherence." These concepts were all related using the Boolean operators. Additional studies were retrieved through references of other articles included in this review.

\section{Inclusion and exclusion criteria}

Observational studies were included in this review if they met the following four criteria (1) participants had type 2 diabetes, (2) used non-insulin anti-diabetic medication, (3) adherence was measured using PDC or MPR, and (4) evaluated a specific patient characteristic and its possible association with adherence. Insulin products were excluded from the study because the Pharmacy Quality Alliance (PQA), which endorses the PDC method, does not consider insulin feasible to measure due to the complexity of measuring adherence to this injectable [3]. However, the injectable glucagon-like peptide-1 (GLP1) agonists are endorsed and therefore were included [9]. The majority of the studies excluded focused on interventions to improve patient's adherence, strategies to measure compliance, and the outcome of non-compliance. These studies were excluded because they did not report adherence as MPR or PDC.

\section{Data Extraction and Analysis}

Studies were first screened based on title and abstract using the described inclusion and exclusion criteria. Then, the relevant studies were evaluated for the characteristics associated with non-adherence to NIAD. The associations were summarized and recorded with their PDC and MPR. The strength of data was then assessed by how the PDC was calculated. The reported PDCs that followed the PQA calculation (for the corresponding year) were considered to be strong. The studies were also evaluated for bias using the Quality Assessment Tool for Observational Cohort and Cross-Sectional Studies by the National Institute of Health (NIH). Studies were rated based on 14 criteria that examined the research question, study population and size, eligibility criteria, level of exposure (e.g. more than one medication), assessment, outcome, follow up, and statistical analysis. Based on these criteria the quality can either be rated as 'good' 'fair' or 'poor.'

\section{Results}

The search result yielded 106 articles. After applying exclusion and inclusion criteria, 25 retrospective cohorts were included. Figure 1 depicts the data abstraction process.(Table 1) shows the detailed description of the adherence studies included in this study. ${ }^{10-34}$ There were 19 studies that used MPR as their compliance measurement [10-29] and 6 studies that used PDC [30-34]. The cut-off value for MPR and PDC was $\geq 80 \%$. The statistical analysis used to assess the effect of various factors on NIAD adherence was a multilevel logistic model because PDC and MPR are continuous variables. There were 13 good studies, 6 fair studies, and 5 poor studies. (Table 1) describes the studies included in this review.

Table 1: Characteristics of the included studies.

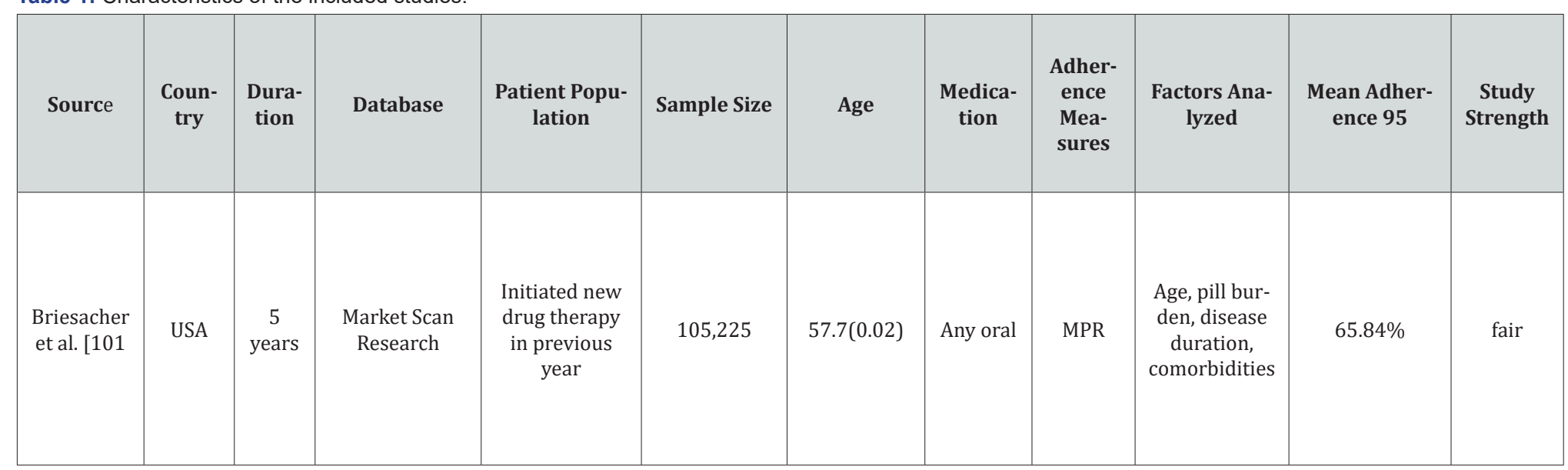




\section{Current Research in Diabetes \& Obesity Journal}

\begin{tabular}{|c|c|c|c|c|c|c|c|c|c|c|c|}
\hline $\begin{array}{c}\text { Calip et al. } \\
\text { [11] }\end{array}$ & USA & $\begin{array}{l}19 \text { yea } \\
\text { rs }\end{array}$ & $\begin{array}{l}\text { Group Health } \\
\text { Cooperative }\end{array}$ & $\begin{array}{l}\text { Women from } \\
\text { COMBO cohort } \\
\text { diagnosed } \\
\text { with early } \\
\text { stage (I,II) } \\
\text { Invasive breast } \\
\text { cancer }\end{array}$ & 509 & 65 & MET, SUA & MPR & $\begin{array}{l}\text { Adherence } \\
\text { before, at, and } \\
\text { after breast } \\
\text { cancer diag- } \\
\text { nosis }\end{array}$ & $\begin{array}{c}\text { Pre-cancer } \\
80.6, \text { post } \\
\text { cancer } 49 \%\end{array}$ & fair \\
\hline $\begin{array}{c}\text { Cho, et al. } \\
\text { [12] }\end{array}$ & $\begin{array}{l}\text { Canada } \\
\text { [British } \\
\text { Colum- } \\
\text { bia) }\end{array}$ & $\begin{array}{c}10 \\
\text { years }\end{array}$ & PharmNet & $\begin{array}{l}\text { South-Asian, } \\
\text { Chinese and } \\
\text { white people } \\
\text { aged } \geq 35 \text { years }\end{array}$ & 37,243 & NA & $\begin{array}{l}\text { higuan } \\
\text { ides, SUA } \\
\text { T2Ds }\end{array}$ & MPR & $\begin{array}{l}\text { race ethnicity, } \\
\text { comorbid } \\
\text { conditions, } \\
\text { socio-eco- } \\
\text { nomic status, } \\
\text { medication } \\
\text { type }\end{array}$ & NA & good \\
\hline $\begin{array}{l}\text { Cohen et al. } \\
\text { [13] }\end{array}$ & USA & - & $\begin{array}{c}\text { 11995EIU } \\
\text { Benefit and } \\
\text { Pensions Fund }\end{array}$ & $\begin{array}{l}\text { mufti-ethnic, } \\
\text { lower income, } \\
\text { Insured adults } \\
\text { in NYC, age } \\
>30\end{array}$ & 526 & $55.5(7.3)$ & Any Oral & MPR & $\begin{array}{c}\text { sex, age, } \\
\text { duration } \\
\text { of disease, } \\
\text { marital status, } \\
\text { income, edu- } \\
\text { cation level }\end{array}$ & NA & poor \\
\hline $\begin{array}{c}\text { Dijk et al. } \\
\text { [14] }\end{array}$ & $\begin{array}{l}\text { Neth- } \\
\text { erlands }\end{array}$ & 1 year & $\begin{array}{l}\text { Dutch gen- } \\
\text { eral practice } \\
\text { registration } \\
\text { database \& } \\
\text { dispensing } \\
\text { registration } \\
\text { database }\end{array}$ & $\begin{array}{l}\text { Patients from a } \\
\text { Dutch general } \\
\text { practice regis- } \\
\text { tration }\end{array}$ & 2,428 & $\begin{array}{l}\text { Nonadherent: } \\
\text { 66.6(13.4); } \\
\text { Adher- } \\
\text { ent:65.8(11.9) }\end{array}$ & $\begin{array}{l}\text { biguan } \\
\text { ides, SUA }\end{array}$ & MPR & $\begin{array}{l}\text { sex, age, edu- } \\
\text { cation, marital } \\
\text { status, cor- } \\
\text { norbidities, } \\
\text { healthcare } \\
\text { contact }\end{array}$ & NA & fair \\
\hline $\begin{array}{c}\text { Egede et al. } \\
\text { [15] }\end{array}$ & USA & $\begin{array}{c}5 \\
\text { years }\end{array}$ & $\begin{array}{l}\text { Linked Vet- } \\
\text { erans Health } \\
\text { Administra- } \\
\text { tion National } \\
\text { Patient Carr } \\
\text { and Pharmacy } \\
\text { Benet. Manage- } \\
\text { ment databases }\end{array}$ & $\begin{array}{l}\text { National } \\
\text { cohort of } \\
\text { veterans }\end{array}$ & 479,248 & $65.7(11.28)$ & Any Oral & MPR & $\begin{array}{l}\text { sex, age, race/ } \\
\text { ethnicity, lo- } \\
\text { cation, marital } \\
\text { status }\end{array}$ & $80.86 . \%$ & poor \\
\hline $\begin{array}{l}\text { Guenetre } \\
\text { et al. [16] }\end{array}$ & $\begin{array}{c}\text { Canada } \\
\text { (Que- } \\
\text { bec) }\end{array}$ & $\begin{array}{c}9 \\
\text { years }\end{array}$ & $\begin{array}{l}\text { Quebec health } \\
\text { insurance } \\
\text { board (RAMQ) }\end{array}$ & $\begin{array}{l}\text { New users of } \\
\text { OADs in Que- } \\
\text { bec in a plan } \\
\text { that covers } \\
\text { ages } 65 \text { or } \\
\text { above, welfare } \\
\text { recipients and } \\
\text { those without } \\
\text { access to } \\
\text { private health } \\
\text { insurance }\end{array}$ & 119,832 & NA & Any Oral & MPR & $\begin{array}{l}\text { age, sex, resi- } \\
\text { dential region, } \\
\text { socioeco- } \\
\text { nomic status, } \\
\text { specialty of } \\
\text { prescriber, } \\
\text { medication, } \\
\text { history of } \\
\text { hospitaliza- } \\
\text { tion, physician } \\
\text { visits, pill } \\
\text { burden }\end{array}$ & $86.3(16.2)$ & poor \\
\hline $\begin{array}{l}\text { Hansen et } \\
\text { al. [17] }\end{array}$ & USA & $\begin{array}{c}3 \\
\text { years }\end{array}$ & $\begin{array}{c}\text { MEDSTAT } \\
\text { MarketScan } \\
\text { Research data- } \\
\text { bases }\end{array}$ & $\begin{array}{c}\text { age } 18-90 \\
\text { years, taking } \\
\text { metformin, } \\
\text { pioglitazone or } \\
\text { a sulfonylu rea, } \\
\geq 2 \text { outpatient } \\
\text { claims or } \geq 1 \\
\text { inpatient claim }\end{array}$ & 108,592 & $54(11)$ & $\begin{array}{c}\text { MET, } \\
\text { SUA, PIO }\end{array}$ & MPR & $\begin{array}{l}\text { Age, sex, in- } \\
\text { surance type, } \\
\text { cornorbidites, } \\
\text { medication } \\
\text { type }\end{array}$ & $\begin{array}{c}\text { metformin: } \\
66.7 \%, \\
\text { pioglitazone: } \\
61.3 \% \text {, } \\
\text { sulfonylurea: } \\
67.5 \%\end{array}$ & good \\
\hline $\begin{array}{c}\text { Haupt et al. } \\
\text { [18] }\end{array}$ & $\begin{array}{l}\text { Swe- } \\
\text { den }\end{array}$ & 1 year & $\begin{array}{l}\text { Swedish pre- } \\
\text { scribed drug } \\
\text { register }\end{array}$ & $\begin{array}{l}\text { Age }>40 \text { years } \\
\text { having } \geq 2 \text { dis- } \\
\text { pensing of one } \\
\text { or more OAD }\end{array}$ & 171,220 & $\begin{array}{c}\text { NA; mode } \\
\text { of age range } \\
\text { was } 75-84 \\
\text { yrs }\end{array}$ & My Oral & MPR & $\begin{array}{l}\text { Age, sex, med- } \\
\text { ication type, } \\
\text { pill burden, } \\
\text { specialty of } \\
\text { prescriber, } \\
\text { indication } \\
\text { written on rx }\end{array}$ & $107 \%$ & good \\
\hline
\end{tabular}




\section{Current Research in Diabetes \& Obesity Journal}

\begin{tabular}{|c|c|c|c|c|c|c|c|c|c|c|c|}
\hline $\begin{array}{c}\text { Jha et al. } \\
\text { [19] }\end{array}$ & USA & $\begin{array}{c}4 \\
\text { years }\end{array}$ & $\begin{array}{l}\text { Medco Health } \\
\text { Solutions }\end{array}$ & $\begin{array}{l}\text { Nationwide } \\
\text { cohort age } \\
>18, \text { majority } \\
\text { covered by } \\
\text { medicare }\end{array}$ & 135,639 & NA & $\begin{array}{l}\text { My Oral } \\
\text { or GIP-1 }\end{array}$ & MPR & $\begin{array}{l}\text { Age, sex, } \\
\text { comorbidities } \\
\text { Income, edu- } \\
\text { cation, race }\end{array}$ & NA & good \\
\hline $\begin{array}{l}\text { Kirkman et } \\
\text { al. [20] }\end{array}$ & USA & 1 year & $\begin{array}{l}\text { Information } \\
\text { warehouse of } \\
\text { Medco Health } \\
\text { Solutions }\end{array}$ & $\begin{array}{l}\text { Patients using } \\
\text { no more than } \\
\text { two oral a } \\
\text { antidiabetic } \\
\text { medications, } \\
\text { patients from } \\
\text { all } 50 \text { states in- } \\
\text { cluding Puerto } \\
\text { Rico, Virgin } \\
\text { islands, Guam, } \\
\text { age >8 }\end{array}$ & 218,384 & $64.9(4.8)$ & $\begin{array}{l}\text { Any Oral } \\
\text { or GLP-1 }\end{array}$ & MPR & $\begin{array}{l}\text { Substance } \\
\text { abuse } \\
\text { disorder, } \\
\text { schizophrenia, } \\
\text { depression, } \\
\text { pill burden, } \\
\text { age, sex, race, } \\
\text { homelessness, } \\
\text { marital status, } \\
\text { copayment, } \\
\text { days supply }\end{array}$ & NA. & 2 \\
\hline $\begin{array}{l}\text { Kreyenbuhl } \\
\text { et al. [21] }\end{array}$ & USA & $\begin{array}{c}2 \\
\text { years }\end{array}$ & $\begin{array}{c}\text { VA's National } \\
\text { Psychosis } \\
\text { Registry }\end{array}$ & $\begin{array}{l}\text { Veterans with } \\
\text { and without } \\
\text { schizophrenia }\end{array}$ & 22,014 & $\begin{array}{c}\text { Diabetes } \\
\text { with schizo- } \\
\text { phrenia: } \\
55.7(10.7) ; \\
\text { diabetes } \\
\text { without } \\
\text { schizophre- } \\
\text { nia } \\
654(106)\end{array}$ & Any Oral & MPR & $\begin{array}{c}\text { Age, sex, race, } \\
\text { marital status, } \\
\text { homelessness, } \\
\text { depression, } \\
\text { PTSD, co- } \\
\text { morbidities, } \\
\text { hospitaliza- } \\
\text { tion in past } \\
\text { year, health } \\
\text { care contact, } \\
\text { mailed } \\
\text { prescriptions, } \\
\text { copay, }\end{array}$ & NA & good \\
\hline $\begin{array}{c}\text { Piette et al. } \\
\text { [22] }\end{array}$ & USA & 1 year & $\begin{array}{l}\text { VA's, Nation, } \\
\text { Psychosis } \\
\text { Registry }\end{array}$ & $\begin{array}{l}\text { Veterans with } \\
\text { schizophrenia, } \\
\text { diabetes, and } \\
\text { hypertension_- } \\
\text { Mostly unmar- } \\
\text { ried males }\end{array}$ & 1,686 & $54.9(10.8)$ & Any Oral & MPR & $\begin{array}{l}\text { Concurrent } \\
\text { schizophrenia, } \\
\text { hypertension, } \\
\text { and days } \\
\text { supply }\end{array}$ & $16 \%$ & good \\
\hline $\begin{array}{l}\text { Rolnick et } \\
\text { al. [23] }\end{array}$ & USA & $\begin{array}{c}2 \\
\text { years }\end{array}$ & $\begin{array}{c}\text { Pharmac } \\
\text { administrative } \\
\text { database }\end{array}$ & $\begin{array}{l}\text { Midwestern, } \\
>18 . \text { with at } \\
\text { least one of } \\
\text { eight comorbid } \\
\text { conditions }\end{array}$ & 4631 & NA & $\begin{array}{l}\text { any oral } \\
\text { or GIP } 1\end{array}$ & MPR & $\begin{array}{l}\text { age, sex; race, } \\
\text { education, } \\
\text { comorbiditie, } \\
\text { pill burden }\end{array}$ & $81 \%$ & good \\
\hline $\begin{array}{l}\text { Rozenfield } \\
\text { et al. [24] }\end{array}$ & USA & $\begin{array}{c}3 \\
\text { years }\end{array}$ & $\begin{array}{c}\text { Pharmacy } \\
\text { claims from a } \\
\text { managed care } \\
\text { plan in oregan }\end{array}$ & $\begin{array}{l}>18 \text { yo living } \\
\text { in Oregon } \\
\text { who recently } \\
\text { initiated oral } \\
\text { antidiabetic }\end{array}$ & 2741 & $54(11)$ & $\begin{array}{c}\text { MET, } \\
\text { SUA, TZD }\end{array}$ & MPR & $\begin{array}{c}\text { medication } \\
\text { class, age, sex, } \\
\text { comorbiditie, } \\
\text { pill burden }\end{array}$ & $\begin{array}{c}\text { rnet- } \\
\text { formin:80.7(21.6), } \\
\text { sulfony- } \\
\text { lurea:11.8(21.7), } \\
\text { TZD: } 82(21.4), \text { total } \\
81.3(21.6)\end{array}$ & poor \\
\hline $\begin{array}{l}\text { Schoen- } \\
\text { thaler et al. } \\
\text { [25] }\end{array}$ & USA & $\begin{array}{c}2 \\
\text { years }\end{array}$ & $\begin{array}{l}\text { physician } \\
\text { orders in elec- } \\
\text { tronic health } \\
\text { records }\end{array}$ & $\begin{array}{l}\text { at least } 4 \text { office } \\
\text { visits in pst } \\
2 \text { years, at } \\
\text { least one oral } \\
\text { hypoglycemic, } \\
\text { ages }>30 \text { and } \\
<75 \text {. majority } \\
\text { were male } \\
\text { and retired, } \\
\text { mean duration } \\
\text { of time with } \\
\text { diabetes: } 5 \\
\text { years }\end{array}$ & 608 & $62.1(9.2)$ & Any Oral & MOO & $\begin{array}{l}\text { age, sex, } \\
\text { duration } \\
\text { of disease, } \\
\text { knowledge of } \\
\text { diabetes, }\end{array}$ & - & good \\
\hline
\end{tabular}




\section{Current Research in Diabetes \& Obesity Journal}

\begin{tabular}{|c|c|c|c|c|c|c|c|c|c|c|c|}
\hline $\begin{array}{c}\text { Tan et al. } \\
{[26]}\end{array}$ & USA & $\begin{array}{c}2 \\
\text { years }\end{array}$ & $\begin{array}{l}\text { MarkeScan } \\
\text { Commercial } \\
\text { Claims and } \\
\text { Encounters } \\
\text { Database }\end{array}$ & $\begin{array}{l}\text { Age 13-64 with } \\
\text { concomitmant } \\
\text { diagnoses of } \\
\text { canoer [breast, } \\
\text { prostate, colon } \\
\text { or long) and } \\
\text { type } 2 \text { diabetes }\end{array}$ & 1918 & 56.7 & Any Oral & MPR & $\begin{array}{c}\text { age sex, } \\
\text { cancer type, } \\
\text { location, } \\
\text { comorbidities, } \\
\text { medication } \\
\text { type, phar- } \\
\text { macy type, } \\
\text { diabetes. com- } \\
\text { plications }\end{array}$ & $60(0.32)$ & poor \\
\hline $\begin{array}{c}\text { Wong et al. } \\
\text { [27] }\end{array}$ & China & $\begin{array}{c}35 \\
\text { years }\end{array}$ & $\begin{array}{l}\text { Clinical Data } \\
\text { Analyses and } \\
\text { Reporting Sys- } \\
\text { tem (CDARS) }\end{array}$ & $\begin{array}{l}\text { Chinese adult } \\
\text { patients. } \\
\text { Majority were } \\
\text { male, fee } \\
\text { payers, lived } \\
\text { in uran areas, } \\
\text { and few co- } \\
\text { morbidities }\end{array}$ & 26,782 & $>60$ & MET, SUA & MPR & $\begin{array}{l}\text { age, se; pay- } \\
\text { ment, service } \\
\text { type, comor- } \\
\text { bidities., drug } \\
\text { class }\end{array}$ & & fair \\
\hline $\begin{array}{l}\text { Zanders et } \\
\text { al. [28] }\end{array}$ & USA & $\begin{array}{c}9 \\
\text { years }\end{array}$ & $\begin{array}{l}\text { Eindhoven } \\
\text { Cancer Regis- } \\
\text { try, PHARMO } \\
\text { Database }\end{array}$ & $\begin{array}{l}\text { Age } 30 \text { years } \\
\text { and over with } \\
\text { any cancer } \\
\text { type (except } \\
\text { melanoma) } \\
\text { and matched } \\
\text { with non- can- } \\
\text { cer controls }\end{array}$ & 15,231 & $\begin{array}{l}67.7(9.8) \\
\text { for cancer } \\
\text { and } 67.5 \\
\text { (9.7) for } \\
\text { controls }\end{array}$ & Any Oral & MOO & $\begin{array}{l}\text { age, sex; can- } \\
\text { cer diagnosis, } \\
\text { cancer type, } \\
\text { medication } \\
\text { class }\end{array}$ & $\begin{array}{c}\text { baseline not } \\
\text { given, average } \\
6.3 \% \text { drop } \\
\text { after cancer di- } \\
\text { agnosis (max:- } \\
15.2 \% \text { ) }\end{array}$ & good \\
\hline $\begin{array}{c}\text { Huber et } \\
212016 \\
1291\end{array}$ & $\begin{array}{l}\text { Swit- } \\
\text { zerland }\end{array}$ & $\begin{array}{c}3 \\
\text { years }\end{array}$ & Helsana Group & $\begin{array}{c}\text { aged } \geq 18 \text { years } \\
\text { with diabetes } \\
\text { and treated } \\
\text { with at least } 1 \\
\text { oral a antidia- } \\
\text { betic }\end{array}$ & 26,713 & $69(12)$ & My Oral & PDC & $\begin{array}{l}\text { sex, age, co- } \\
\text { pay, preceding } \\
\text { hospitaliza- } \\
\text { tion, dispens- } \\
\text { ing channel, } \\
\text { number of } \\
\text { comorbidities, } \\
\text { drug therapy, }\end{array}$ & $68 \%$ & fair \\
\hline $\begin{array}{c}\text { Huber et al. } \\
\text { [30] }\end{array}$ & $\begin{array}{l}\text { Swit- } \\
\text { zerland }\end{array}$ & $\begin{array}{c}3 \\
\text { years }\end{array}$ & Helsana Group & $\begin{array}{c}\text { aged } \geq 8 \text { years } \\
\text { with diabetes } \\
\text { and treated } \\
\text { with at least } 1 \\
\text { oral a Antidia- } \\
\text { betic }\end{array}$ & 26,722 & $69(12)$ & My Oral & PDC & $\begin{array}{l}\text { sex, age, cope, } \\
\text { preceding } \\
\text { hospitaliza- } \\
\text { tion, number } \\
\text { of comorbid- } \\
\text { ities, drug } \\
\text { therapy, } \\
\text { dispensing } \\
\text { channel }\end{array}$ & $70 \%$ & good \\
\hline $\begin{array}{c}\text { Juarez et al. } \\
\text { [31] }\end{array}$ & $\begin{array}{l}\text { USA } \\
\text { (Hawa- } \\
\text { li) }\end{array}$ & $\begin{array}{c}4 \\
\text { years }\end{array}$ & $\begin{array}{l}\text { Hawali health } \\
\text { plan }\end{array}$ & $\begin{array}{l}\text { adult patients } \\
\text { with diabetes } \\
\text { enrolled in } \\
\text { health plan in } \\
\text { Hawali for } 4 \\
\text { years }\end{array}$ & 23,450 & $60(13)$ & Any Oral & PDC & $\begin{array}{c}\text { age, sex, } \\
\text { number of } \\
\text { comorbidities } \\
\text { and type, } \\
\text { ethnicity, pill } \\
\text { burden }\end{array}$ & $55 \%$ & good \\
\hline $\begin{array}{c}\text { Stuart et al. } \\
\text { [32] }\end{array}$ & USA & 1 year & $\begin{array}{c}\text { Chronic Con- } \\
\text { ditions Data } \\
\text { Warehouse } \\
\text { (CCW) }\end{array}$ & $\begin{array}{l}5 \% \text { random } \\
\text { sample of } \\
\text { Medicare part } \\
\text { D beneficiaries } \\
\text { with type } 2 \\
\text { diabetes. Pa- } \\
\text { tients who had } \\
\text { newly diag- } \\
\text { nosed cancer } \\
\text { wet compared } \\
\text { to cancer-free } \\
\text { control }\end{array}$ & 32755 & NA & $\begin{array}{l}\text { Any } \\
\text { Oral and } \\
\text { GLP-1 } \\
\text { agonists }\end{array}$ & PDC & $\begin{array}{l}\text { cancer diag- } \\
\text { nosis }\end{array}$ & $\begin{array}{c}\text { Pre-cancer: } \\
\text { 80.8, post } \\
\text { cancer: } 73.8\end{array}$ & good \\
\hline
\end{tabular}




\section{Current Research in Diabetes \& Obesity Journal}

\begin{tabular}{|c|c|c|c|c|c|c|c|c|c|c|c|}
\hline $\begin{array}{c}\text { Turret et } \\
\text { al. [33] }\end{array}$ & USA & $\begin{array}{c}3 \\
\text { years }\end{array}$ & $\begin{array}{l}\text { Truven Health } \\
\text { MarketScan } \\
\text { Commercial } \\
\text { Claims and } \\
\text { Encounters } \\
\text { and Medicare } \\
\text { Supplemental } \\
\text { and Coordina- } \\
\text { tion of Benefits } \\
\text { Databases }\end{array}$ & $\begin{array}{l}\text { aged } \geq 8 \text { years } \\
\text { with diabetes } \\
\text { who represent } \\
\text { nationally } \\
\text { comercial- } \\
\text { ly insured } \\
\text { population } \\
\text { who have both } \\
\text { medicare and } \\
\text { supplemental } \\
\text { coverage }\end{array}$ & 113,449 & $60.7(12.6)$ & Any Oral & PDC & $\begin{array}{l}\text { age, pill bur- } \\
\text { den, disease } \\
\text { duration, dos- } \\
\text { ing regimen, } \\
\text { sex, copayr- } \\
\text { nent }\end{array}$ & $75 \%$ & fair \\
\hline $\begin{array}{c}\text { Yang et al. } \\
{[34]}\end{array}$ & USA & 1 year & $\begin{array}{c}\text { Medicare Part } \\
\text { D Prescription } \\
\text { claims data }\end{array}$ & $\begin{array}{l}\text { Medicare Part } \\
\text { D enrollees } \\
\text { from } 6 \text { dis- } \\
\text { persed states } \\
\text { (Alabama, Cali- } \\
\text { fornia, Florida, } \\
\text { Mississpi, New } \\
\text { York, Ohio) }\end{array}$ & $1,888,632$ & $71.6(11.6)$ & My Oral & PDC & $\begin{array}{l}\text { age, sex, race, } \\
\text { comorbidities }\end{array}$ & $65 . \%$ & good \\
\hline
\end{tabular}

All patient populations in the studies had T2DM and used at least one NIAD. The majority of the studies measured adherence to any oral hypoglycemic $[10,13,18,21,22,25,26,28-31,33,34]$, while 4 broadened their coverage to include GLP-1 agonists $[19,20,23,32]$, and 7 only included specific oral hypoglycemics $[11,12,14,17,24,27,32]$. The average age of the study population ranged from $56-84[13,18]$ The studies were performed in various countries including the United States [10,11,13,15,17-26,31-34], Canada [12,16], Netherlands [14], Sweden [18], China [27], and Switzerland [29,30]. The study duration ranged from 1 year to 19 years $[11,14,18,20,22,32,34]$.The sample size ranged from 509 to
$1,888,682$ people. ${ }^{11,34}$. Some unique patient populations studied were those with cancer $[11,26,28,32]$, schizophrenia [21,22], and veterans[15,21,22].

\section{Factors associated with medication adherence}

The factors contributing to MNA were grouped based on the pre-established dimensions of medication adherence found by WHO (Table 2). These dimensions include: 1) social and economic-related factors; 2) health care team and system-related factors; 3) condition-related factors; 4) therapy related factors; and 5) patient related factors [7].

Table 2: Factors contributing to medication non-adherence categorized based on pre-established dimensions of medication adherence found by world health organization (WHO).

\begin{tabular}{|c|c|c|c|}
\hline Factors & $\begin{array}{l}\text { Studies with Positive Asso- } \\
\text { ciation with Adherence }\end{array}$ & $\begin{array}{l}\text { Studies with Negative Association } \\
\text { with Adherence }\end{array}$ & $\begin{array}{l}\text { Studies with no Significant Associa- } \\
\text { tion with Adherence }\end{array}$ \\
\hline \multicolumn{4}{|l|}{ Social and economic-related factors } \\
\hline Increasing age & $\begin{array}{c}15[10,16,17,18,19,20,23,24, \\
26,27,29,30,31,33,34]\end{array}$ & $2[15,28]$ & $2[21,25]$ \\
\hline Gender, male & $\begin{array}{c}10[15,19,20,23,25,29,30,31 \\
, 33,34]\end{array}$ & $3[13,18,27]$ & $5[14,16,21,24,26]$ \\
\hline Non-caucasian & & $8[12,13,15,19,21,23,31,34]$ & \\
\hline Higher Education & $2[19,20]$ & & $2[14,23]$ \\
\hline Low income status & $2[12,16]$ & $2[19,20]$ & $1[23]$ \\
\hline Homelessness & & $1[21]$ & \\
\hline Rural resident & $2[15,16]$ & & $2[26,27]$ \\
\hline Married & $1[15]$ & $1[14]$ & $1[21]$ \\
\hline Low copayment & $4[20,21,30,33]$ & & $1[27,34]$ \\
\hline \multicolumn{4}{|l|}{$\begin{array}{l}\text { Health care team and system-relat- } \\
\text { ed factors }\end{array}$} \\
\hline $\begin{array}{l}\text { shared decision making with } \\
\text { physician }\end{array}$ & $1[25]$ & & \\
\hline $\begin{array}{l}\text { Increased time spent with physi- } \\
\text { cian }\end{array}$ & $1[25]$ & & \\
\hline
\end{tabular}




\section{Current Research in Diabetes \& Obesity Journal}

\begin{tabular}{|c|c|c|c|}
\hline Indication written on the rx & $1[18]$ & & \\
\hline GP as Initial prescriber & $2[16,20]$ & & $2[18,24]$ \\
\hline Average days' supply >60 & $1[22]$ & & $1[21]$ \\
\hline Mailed prescriptions. & $3[20,21,26]$ & $1[30]$ & \\
\hline \multicolumn{4}{|l|}{ dispensing channel } \\
\hline \multicolumn{4}{|l|}{ Condition-related factors } \\
\hline hospitalization in past year & $1[16]$ & $3[21,29,30]$ & \\
\hline Long duration of diabetes & $4[10,13,20,33]$ & & \\
\hline $\begin{array}{l}\text { Severity of diabetes related com- } \\
\text { plications }\end{array}$ & & $2[15,25]$ & $1[26]$ \\
\hline Number of comorblditles & $7[12,16,24,27,29,30,31]$ & & $2[14,26]$ \\
\hline frequent healthcare visits & $3[11,16,27]$ & $4[17,21,23,34]$ & $1[14]$ \\
\hline Cancer Diagnosis & & $3[26,28,32]$ & \\
\hline Cancer type & & more severe: 213,32 & $1[26]$ \\
\hline Coronary Artery Disease & & $1[31]$ & \\
\hline Congestive heart failure & & $1[31]$ & \\
\hline hypertension & $1[22]$ & & \\
\hline Substance Abuse Disorder & & $1[21]$ & \\
\hline Schizophrenia & $1[21]$ & $1[22]$ & \\
\hline Depression & & $1[21]$ & \\
\hline \multicolumn{4}{|l|}{ Therapy-related factors } \\
\hline Metformin & $5[12,16,18,26,29]$ & & $3[14,24,271$ \\
\hline Sulfonylurea & $2[17,18]$ & $4[12,16,26,28]$ & $3[14,24,27]$ \\
\hline Other medication type & & $5[12,16,17,18,261$ & $1[24]$ \\
\hline Pill burden & $8[10,16,20,21,24,29,30,33]$ & $4[11,14,23,26]$ & $2[14,27]$ \\
\hline \multicolumn{4}{|l|}{ Patient-related factors } \\
\hline $\begin{array}{l}\text { increased diabetes related knowl- } \\
\text { edge }\end{array}$ & & $1[25]$ & \\
\hline agree dependent on medications & & $1[25]$ & \\
\hline
\end{tabular}

\section{Social and economic-related factors}

Many studies supported that increasing age $[10,16-20,23,24,26,27,29-31,33,34], \quad$ and male gender[15,19,20,23,25,29,30,31,33,34], were the most associated with NIAD adherence. Non-Caucasian race consistently showed decreased adherence compared to Caucasians, except for the Japanese [31]. The races evaluated that showed lower adherence were Hispanic [13,15,23,34], non-Hispanic black $[13,15,19,21,23,34]$

, Chinese [12,27], South-Asian descent, [12] and Filipino [31]. The associations between NIAD adherence with income, copayment, education level, marital status, and rural residence were equivocal due to the number and quality of studies that supported the associations.

\section{Health care team and system-related factors}

The most prevalent health system-related factors that were observed were the specialty of the prescriber and the use of mailed prescriptions. Patients are more likely to be adherent to their NIAD if their initial prescriber was a general practitioner (GP) or endocrinologist, rather than another specialist such as an internist $[16,20]$.

\section{Condition-related factors}

Conditions consistently associated with MNA were a hospitalization in the past year $[21,29,30]$, infrequent physician visits [11,16,27], and a diagnosis of cancer [26,28,32]. However, Guénette et al found that when the physician consultations exceeded 18, the odds of adherence decreased (OR:0.93, 95\% CI: $[\mathrm{xx}, \mathrm{xx}])[16]$. Also, the type of cancer diagnosis is also significant 
as more severe cancers lead to more profound drop in MPR or PDC than with breast or prostate cancer $[28,32]$. The association between NIAD adherence with duration of the disease as well as the presence of comorbidities is difficult because there are multiple studies that have found positive and negative relationships with both these conditions. Huber et al found an interesting relationship with the number of comorbidities and proportion of adherent patients. In a sample 26,713 patients, the proportion of adherent patients with $0-1,2-4$, and $\geq 5$ comorbidities was $24 \%$, $58.4 \%$, and $17.6 \%$, respectively. ${ }^{29}$ This suggests that having $2-4$ comorbidities is associated with increased adherence.

\section{Therapy-related factors}

In regard to medication regimen, patients prescribed metformin $[12,16,18,26,29]$, and who have a higher pill burden are more likely to be adherent $[10,16,20,21,24,29,30,33]$.

\section{Patient-related factors}

The only study that analyzed WHO defined patient relatedfactors was a study by Schoenthaler et al,. Interestingly, they found a negative relationship between patients' diabetes-related knowledge and adherence [25].

\section{Discussion}

This SR identified studies reporting associations with MNA in T2DM patients using NIAD. Previous SRs have studied MNA factors, but this is the first SR that utilized MPR and PDC to characterize MNA and to group the factors based the five WHO dimensions of MNA [7]. WHO recognized that cost of care was the primary social and economic related factor associated with MNA. This study identified only two studies that supported this finding and two studies that did not $[12,16,19,20]$. Both articles that showed a positive association were graded as 'poor' quality and therefore based on the strength of evidence this not considered a social and economic related factor. Social and economic relatedfactors that were not previously analyzed were: age[10,1521,23-31,33,34],gender [13-16,18-21,23-27,29-31,33,34], race/ ethnicity, [12,13,15,19,21,23,31,34] education level [14,19,20,23], income status $[12,16,19,20,23]$, area of residence $[15,16,26,27]$, homelessness, [21] and marital status [15,21]. Of these factors listed, the highest strength of evidence based on the number and quality of studies were the first three. The clinical implication of racial minorities being MNA is profound. Poor glycemic control achieved by racial minorities due to NIAD MNA has shown a strong association with increased mortality $[35,36]$. It is postulated that MNA in racial minorities is attributed to language barriers [37], low health literacy [38], and preference of alternative medicine [39]. The poor adherence observed in the Asian population $[12,23,31]$, except for the Japanese [31] may have been primarily influenced by use of traditional medicine. Wilson et al found that $33 \%$ of south Asians with T2DM preferentially used alternative medicine for glycemic control [40]. Therefore, it is imperative that healthcare providers (HCPs) inquire about alternative medicine and work with the patients to determine which supplements can be used to compliment prescribed therapy. It is also important that HCPs recognize that younger patients, especially under 54 years old, are more likely to be MNA [16]. The only health care team and system related factors that WHO identified was a poor relationship between the patient and physician [7]. Only one study was found that supported this association, therefore an association cannot be determined [25]. The only major factor observed for this dimension was mailed prescriptions $[20,21,26]$. The association may be attributed to increased day's supply, lower price, and convenient telephone consultations provided by the service [41].

The condition-related factors associated with MNA identified by WHO were depression and increased duration of disease [7]. No articles that met the inclusion criteria for this SR analyzed the association with depression. However, this association was found in studies that included insulin $[42,43]$. In contrast to WHO's findings, this SR presents that adherence may be associated with longer duration of diabetes rather than shorter $[10,13,20,33]$. However, this factor needs further investigation as this information is unavailable to some studies [15,25], and there was one good and one poor quality study that supported the contrary $[15,25]$. The most clinically useful information for HCPs regarding condition-related factors would be the association of MNA with cancer diagnosis [26,28,32] and comorbidities $[12,16,17,21,23,24,26,27,29-31]$, MNA following a cancer diagnosis, especially in patients with a worse prognosis[28,32], was consistent with the finding that major life events with psychological stressors leads to a significant drop anti-diabetic medication adherence [44]. However, it is imperative that cancer patients continue their NIAD, especially metformin, as it has been shown to improve breast cancer prognosis as well as inhibit tumor growth [45-47]. There may be a relationship between fewer comorbidities, younger age, newer to diabetes, and on fewer medications as described by Kirkman et al, [20]. Venturini suggested that this is because younger patients have less comorbidities and sequentially less complex therapies and therefore do not perceive their health to be dependent on their medicine [48]. Therefore, this population should be especially targeted for interventions of adherence. Regarding therapyrelated factors, WHO found that MNA was associated with complex treatment and increased pill burden [7], which is contrary to the relationship described by Kirkman and Venturini [20,47]. Although there were studies that showed decreased adherence with increased pill burden $[11,18,26]$, there were twice the number of studies, which observed the contrary[10,16,20,21,24,29,30,33], and three of which were higher quality $[10,21,30]$. Therefore, HCP should be especially cognizant of the potential of MNA with patients who are on monotherapy. The patient-related factors identified by WHO that were associated by MNA in diabetic patients were depression, stress, alcohol abuse, and poor self-esteem/self- 
efficacy [7]. Arguably, these factors should have been placed under condition-related factors, as the author explains that condition related factors include comorbidities, such as depression, HIV, and substance abuse. The patient-related factors consist of attitudes, beliefs, and perceptions [7]. Fitting this criterion, the only patient related factors were reported in a study by Schoenthaler et al, [25], which showed MNA was associated with increased diabetes related knowledge and decreased perceived dependence on their medication [25]. The latter is consistent with the medication dependence conclusion made by Venterini et al, [48] However, HCP should be careful when interpreting these associations as this study was only graded as fair due to the sample size and low external validity.

There are several limitations to this study. Firstly, insulin was not included in this SR and there was a disproportionately lower number of studies that included GLP-1 agonists. This is significant because there are differences in medication-use behaviors for oral vs. injectable drugs [26]. Secondly, the degree a factor was associated with MNA could not be compared across studies because some studies reported their results with odds ratios, in text, or the percentage of MPR or PDC achieved. Thirdly, there was clearly a correlation between concomitant medications, age, and health status but the exact relationship was not determined. Fourthly, the data can only show associations but not causality. Fifthly, there are assumptions that are made with claims data that do not always hold true, which include: the NIAD were picked up in a timely manner, the NIAD were used by the patient and intended patient only, and that the prescriptions filled were accurately captured in the data set [26]. Finally, the PDC is preferred over MPR for measuring adherence, which is the standard measurement for CMS [49]. However, there were not enough studies that used PDC to measure adherence which is why studies using MPR were also included.

\section{Conclusion}

The SR showed that MNA to NIAD as measured by PDC and MPR is associated with younger age, racial minorities, female gender, fewer comorbidities, diagnosis of cancer and decreased pill burden. More studies are needed which include GLP-1 agonists, use PDC to measure adherence, and examine the relationship between age, comorbidities, and pill burden.

\section{References}

1. (2011) Centers for Disease Control and Prevention. National diabetes fact sheet: national estimates and general information on diabetes and prediabetes in the United States, 2011. Atlanta, GA: U.S. Department of Health and Human Services, Centers for Disease Control and Prevention.

2. (1997-2011) Centers for Disease Control and Prevention. Age-adjusted -percentage of adults with diabetes using diabetes medication, by type of medication, United States.

3. Stolpe S, Kroes MA, Webb N, Hons B, Wisniewski T (2016) A systematic review of Insulin adherence measures in patients with diabetes. J
Manag Care Spec Pharm 22(11): 1224-1246.

4. Saundankar V, Peng X, Fu H, Ascher-Svanum H, Rodriguez A et al. (2016) Predictors of change in adherence status from 1 year to the next among patients with type 2 diabetes mellitus on oral antidiabetes drugs. J Manag Care Spec Pharm 22(5): 467-482.

5. Zomahoun H, Moisan J, Lauzier S, Guillaumie L, Grégoire J-P, et al. (2016) Predicting noninsulin antidiabetic drug adherence using a theoretical framework based on the theory of planned behavior in adults with type 2 diabetes: A prospective study. Medicine 95(15): e2954.

6. Nichols G, Rosales A, Kimes T, Tunceli K, Kurtyka K, et al. (2015) Impact on glycated haemoglobin of a biological response-based measure of medication adherence. Diabetes, Obesity and Metabolism 17(9): 843848.

7. Sabate'E (2003) Adherence to long-term therapies: evidence for action. Geneva, Switzerland: World Health Organization.

8. Sapkota S, Brien J, Greenfield J, Aslani P (2015) A systematic review of interventions addressing adherence to anti-diabetic medications in patients with type 2 diabetes-components of interventions. PLOoS ONE 10(2): e0118296.

9. (2017) Health Insurance Marketplace. 2017 quality rating system measure technical specifications.

10. Briesacher B, Andrade S, Fouayzi H, Chan KA (2008) Comparison of drug adherence rates among patients with seven different medical conditions. Pharmacotherapy 28(4): 437-443.

11. Calip G, Hubbard R, Stergachis A, Malone KE, Gralow J, et al. (2015) Adherence to oral diabetes medications and glycemic control during and following breast cancer treatment. Pharmacoepidemiol Drug Saf 24(1): 75-85.

12. Chong E, Wang H, King Shier KM, Quan H, Rabi D, et al. (2014) Prescribing patterns and adherence to medication among South-Asian, Chinese and white people with type 2 diabetes mellitus: a populationbased cohort study. Diabet Med 31(12): 1586-1593.

13. Cohen H, Shmukler C, Ullman R, Rivera C, Walker E (2010) Measurements of medication adherence in diabetic patients with poorly controlled HbA(1c). Diabet Med 27(2): 210-216.

14.van Dijk L, Heerdink E, Somai D, van Dulmen S, Sluijs EM et al. (2007) Patient risk profiles and practice variation in nonadherence to antidepressants, antihypertensives and oral hypoglycemics. BMC Health Serv Res 7: 51.

15. Egede LE, Gebregziabher M, Hunt KJ, Axon RN, Echols C, et al. (2011) Regional, geographic, and ethnic differences in medication adherence among adults with type 2 diabetes. Ann Pharmacother 45(2): 169-178.

16. Guenette L, Moisan J, Breton MC, Sirois C, Grégoire JP (2013) Difficulty adhering to antidiabetic treatment: factors associated with persistence and compliance. Diabetes Metab 39(3): 250-257.

17. Hansen R, Farley JF, Droege M, Maciejewski M (2010) A retrospective cohort study of economic outcomes and adherence to monotherapy with metformin, pioglitazone, or a sulfonylurea among patients with type 2 diabetes mellitus in the United States from 2003 to 2005. Clin Ther 32(7): 1308-1319.

18. Haupt D, Weitoft GR, Nilsson JL (2009) Refill adherence to oral antihyperglycaemic drugs in Sweden. Acta Diabetol 46(3): 203-208.

19. Jha AK, Aubert RE, Yao J, Teagarden JR, Epstein RS (2012) Greater adherence to diabetes drugs is linked to less hospital use and could save nearly $\$ 5$ billion annually. Health Aff (Millwood) 31(8): 18361846. 
20. Kirkman MS, Rowan-Martin MT, Levin R, Fonseca VA, Schmittdiel JA, et al. (2015) Determinants of adherence to diabetes medications: findings from a large pharmacy claims database Diabetes Care 38(4): 604-609.

21. Kreyenbuhl J, Dixon LB, McCarthy JF, Soliman S, Ignacio RV (2010) Does adherence to medications for type 2 diabetes differ between individuals with vs without schizophrenia? Schizophrenia Bulletin 36(2): 428-435.

22. Piette JD, Heisler M, Ganoczy D, McCarthy JF, Valenstein M (2007) Differential medication adherenceamong patients with schizophrenia and comorbid diabetes and hypertension. Psychiatric Services 58(2): 201-212.

23. Rolnick SJ, Pawloski PA, Hedblom BD, Asche SE, Bruzek RJ (2013) Patient characteristics associated with medication adherence. Clin Med Res 11(2): 54-65.

24. Rozenfeld Y, Hunt JS, Plauschinat C, Wong KS (2008) Oral antidiabetic medication adherence and glycemic control in managed care. Am J Manag Care 14(2): 71-75.

25. Schoenthaler AM, Schwartz BS, Wood C, Stewart WF (2012) Patient and physician factors associated with adherence to diabetes medications. Diabetes Educ 38(3): 397-408.

26. Tan X, Feng X, Chang J, Higa G, Want L (2016) Oral antidiabetic drug use and associated health outcomes in cancer patients. J Clin Pharm Ther 41(5): 524-531.

27. Wong MC1, Kong AP, So WY, Jiang JY, Chan JC, et al. (2011) Adherence to oral hypoglycemic agents in 26,782 Chinese patients: a cohort study. J Clin Pharmacol 51(10): 1474-1482.

28. Zanders MM, Haak HR, van Herk-Sukel MP, van de Poll-Franse LV, Johnson JA (2015) Impact of cancer on use of glucose-lowering drug treatment in individuals with diabetes: potential mechanisms. Diabetologia 58(6): 951-960.

29. Huber CA, Rapold R, Brüngger B, Reich O, Rosemann T (2016) One-year adherence to oral antihyperglycemic medication and risk prediction of patient outcomes for adults with diabetes mellitus. Medicine (Baltimore) 95(26): e3994.

30. Huber CA, Reich O (2016) Medication adherence in patients with diabetes mellitus: does physician drug dispensing enhance quality of care? Evidence from a large health claims database in Switzerland Patient Prefer Adherence 10: 1803-1809.

31. Juarez D, Tan C, Davis J, Mau M (2013) Factors Affecting sustained medication adherence and its impact on health care utilization in patients with diabetes. J Pharm Health Serv Res 4(2): 89-94.

32. Stuart BC, Davidoff AJ, Erten MZ (2015) Changes in medication management after a diagnosis of cancer among Medicare beneficiaries with diabetes. J Oncol Pract 11(6):429-434.

33. Tunceli K, Zhao C, Davies MJ, Brodovicz KG, Alexander CM, et al (2015) Factors associated with adherence to oral antihyperglycemic monotherapy in patients with type 2 diabetes. Patient Prefer Adherence 28(9): 191-197.

34. Yang Y, Thumula V, Pace PF, Banahan BF, Wilkin NE, et al. (2009) Predictors of medication nonadherence among patients with diabetes in Medicare Part D programs: a retrospective cohort study. Clin Ther 31(10): 2178-2188.
35. Hunt KJ, Gebregziabher M, Lynch CP, Echols C, Mauldin PD, et al. (2013) Impact of diabetes control on mortality by race in a national cohort of veterans. Ann Epidemiol 23(2):74-79.

36. Egede LE, Lynch CP, Gebregziabher M, Hunt KJ, Echols C, et al. (2012) Differential impact of longitudinal medication non-adherence on mortality by race/ethnicity among veterans with diabetes. J Gen Intern Med 28(2): 208-215.

37. Lopez Q Berry EM, Neumark Y (2009) Limited English proficiency is a barrier to receipt of advice about physical activity and diet among Hispanics with chronic diseases in the United States. J Am Diet Assoc 110(10): 1769-1774.

38. King KM, Mathers CD, Sanguins J (2005) Ethnocultural affiliation, gender, and cardiovascular disease risk management. Can J Cardiovasc Nurs 15(2): 10-16.

39. Pieroni A, Sheikh QZ, Ali W, Torry B. (2008) Traditional medicines used by Pakistani migrants from Mirpur living in Bradford, Northern England. Complement Ther Med 16(2): 81-86.

40. Wilson E, Wardle EV, Chandel P, Walford S (1993) Diabetes education: an Asian perspective. Diabet Med 10(2): 177-180.

41. Wu J, Davis-Ajami ML, Noxon V (2015) Patterns of use and expenses associated with mail-service pharmacy in adults with diabetes. J Am Pharm Assoc 55(1): 41-51.

42. Balkrishnan R1, Rajagopalan R, Camacho FT, Huston SA, Murray FT, et al. (2003) Predictors of Medication Adherence and Associated Health Care Costs in an Older Population with Type 2 Diabetes Mellitus: A Longitudinal Cohort Study. Clin Ther 25(11): 2958-2971.

43. Axon RN, Gebregziabher M, Hunt KJ, Lynch CP, Payne E, et al. (2016) Comorbid depression is differentially associated with longitudinal medication nonadherence by race/ethnicity in patients with type 2 diabetes. Medicine (Baltimore) 95(2): e3989.

44. Peyrot M, McMurry JF, Kruger DF (1999) A biopsychosocial model of glycemic control in diabetes: stress, coping and regimen adherence. J Health Soc Behav 40(2): 141-158.

45. Chlebowski RT, McTiernan A, Wactawski-Wende J, Manson JE, Aragaki AK, et al. (2012) Diabetes, metformin, and breast cancer in postmenopausal women. J Clin Oncol 30(23): 2844-2852.

46. Hirsch HA, Iliopoulos D, Tsichlis PN, Struhl K (2009) Metformin selectively targets cancer stem cells and acts together with chemotherapy to blocks tumor growth and prolong remission. Cancer Res 69(19): 7507-7511.

47. Hirsch HA, Iliopoulos D, Joshi A, Zhang Y, Jaeger SA, et al. (2010) Transcriptional signature and common gene networks link cancer with lipid metabolism and diverse human diseases. Cancer Cell17(4): 348-361.

48. Venturini F, Nichol MB, Sung JC, Bailey KL, Cody M, et al. (1999) Compliance with sulfonylureas in a health maintenance organization: a pharmacy record-based study. Ann Pharmacother 33(3): 281-288.

49. http://pqaalliance.org/resources/adherence.asp. 


Your next submission with Juniper Publishers
will reach you the below assets
- Quality Editorial service
- Swift Peer Review
- Reprints availability
- E-prints Service
- Manuscript Podcast for convenient understanding
- Global attainment for your research
- Manuscript accessibility in different formats
( Pdf, E-pub, Full Text, Audio)
- Unceasing customer service
Track the below URL for one-step submission
https://juniperpublishers.com/online-submission.php

